involved in prostaglandin- $\mathrm{E}_{2}\left(\mathrm{PGE}_{2}\right)$ synthesis, were also upregulated in Th17-RASF cultures. This was associated with a dramatic increase of $\mathrm{PGE}_{2}$ production in Th17-RASF cultures compared to Th1-RASF cultures. Treatment of Th17-RASF cultures with celecoxib, an inhibitor of cyclo-oxygenase-2 (COX-2) activity, resulted in a significant decrease of IL-17A, but not of IFN $\gamma$ and TNF $\alpha$ production. Furthermore, the decrease in IL-17A production caused by celecoxib treatment was much more pronounced compared to neutralising IL-1 and IL- 6 activity. Flow cytometry revealed a decrease in Th17 cells in celecoxib treated co-cultures, while no effects on Th1 or Th2 cells were found. Moreover, celecoxib treatment significantly inhibited the pro-inflammatory loop as less induction of IL-6, IL- 8 and matrix metalloproteinase- 3 production was observed in the Th17-RASF co-cultures.

Conclusions These findings show the critical role of the COX/ $\mathrm{PGE}_{2}$ pathway in the autocrine IL-17A production upon Th17 and synovial fibroblast interaction. Inhibition of this pathway down-regulates the pro-inflammatory feedback loop induced by Th17-RASF interaction leading to less production of proinflammatory cytokines and destructive mediators.

\title{
24 THE COX/PROSTAGLANDIN-E2 PATHWAY IS CRITICAL FOR AUTOCRINE IL-17A PRODUCTION BY TH17 CELLS UPON SYNOVIAL FIBROBLAST INTERACTION
}

Sandra M J Paulissen, ${ }^{1,2}$ Jan Piet van Hamburg, ${ }^{1,2}$ Nadine Davelaar, ${ }^{1,2}$ Patrick S Asmawidjaja, ${ }^{1,2}$ Johanna MW Hazes, ${ }^{1}$ Erik Lubberts ${ }^{1,2}{ }^{1}$ Department of Rheumatology, Erasmus MC, University Medical Center Rotterdam, Rotterdam, The Netherlands; 2Department of Immunology, Erasmus MC, University Medical Center Rotterdam, Rotterdam, The Netherlands

\subsection{6/annrheumdis-2011-201230.24}

Background and objectives Recently, the authors have shown that Th17, but not Th1 cells, from patients with early rheumatoid arthritis (RA) are potent activators of RA synovial fibroblasts (RASF) resulting in autocrine IL-17A production. This autocrine IL-17A production by Th17 cells is critical for the perseverance of the pro-inflammatory loop, but the mechanism underlying the autocrine IL-17A induction is unclear.

Objective To investigate the mechanism responsible for the autocrine IL-17A induction upon Th17-RASF interaction.

Materials and methods CD4+CD45RO+CCR6+ (Th17) and CD4+CD45RO+CCR6- (Th1) cells were isolated by FACS sorting from healthy controls and early RA patients. These cells were co-cultured with RASF, in the presence of neutralising antibodies directed against soluble IL-6R (anti-sIL-6R) and/or IL-1 $\beta$, and celecoxib. Gene expression profiles were generated and supernatant was collected for cytokine analyses by ELISA.

Results Gene expression analyses revealed that the genes encoding for IL- 6 and IL-1 $\beta$ were up-regulated in Th17-RASF cultures. These data were confirmed by ELISA and Q-PCR, respectively. Since IL-1 $\beta$ and IL- 6 may be involved in IL-17/ Th17 polarisation the authors examined the contribution of these cytokines on the autocrine IL-17A loop. Blockade of IL-1 $\beta$ and IL- 6 activity, but not TNF $\alpha$ significantly suppressed IL-17A production in the co-culture, but the inhibitory effects were limited. Of note, the combination of anti-IL-1 $\beta$ and anti-sIL-6R markedly suppressed IL-17F. No effects were found on IFN $\gamma$. Interestingly, the genes encoding for cyclo-oxygenase-2 (COX-2) and prostaglandin-E-synthase (PTGES), which are 